\title{
New tacrine-derived AChE/BuChE inhibitors: synthesis and biological evaluation of 5- amino-2-phenyl-4H-pyrano[2,3-b]quinoline-3-carboxylates
}

Mohammad Eghtedari ${ }^{\mathrm{a}}$, Yaghoub Sarrafi ${ }^{\mathrm{a}^{* *}}$, Hamid Nadri ${ }^{\mathrm{b}}$, Mohammad Mahdavi ${ }^{\mathrm{c}}$, Alireza Moradi $^{\text {b }}$, Farshad Homayouni Moghadam ${ }^{\mathrm{d}}$, Saeed Emami ${ }^{\mathrm{e}}$, Loghman Firoozpour ${ }^{\mathrm{c}}$, Ali Asadipour $^{\mathrm{f}}$, Omid Sabzevari ${ }^{\mathrm{g}}$, Alireza Foroumadi ${ }^{\mathrm{f}} \mathrm{h}_{*}$

${ }^{a}$ Faculty of Chemistry, University of Mazandaran, Babolsar, Iran

${ }^{b}$ Department of Medicinal Chemistry, Faculty of Pharmacy, Shahid Sadoughi University of Medical Sciences, Yazd, Iran

${ }^{c}$ Drug Design and Development Research Center, Tehran University of Medical Sciences,

Tehran, Iran

${ }^{d}$ Department of Cellular Biotechnology, Cell Science Research Center, Royan Institute for Biotechnology, ACECR, Isfahan, Iran.

${ }^{e}$ Department of Medicinal Chemistry and Pharmaceutical Sciences Research Center, Faculty of Pharmacy, Mazandaran University of Medical Sciences, Sari, Iran

${ }^{f}$ Department of Medicinal Chemistry, Faculty of Pharmacy and Neuroscience Research Center, Institute of Neuropharmacology, Kerman University of Medical Sciences, Kerman, Iran

${ }^{g}$ Department of Pharmacology and Toxicology, Faculty of Pharmacy, Tehran University of Medical Sciences, Tehran, Iran

${ }^{h}$ Department of Medicinal Chemistry, Faculty of Pharmacy and Pharmaceutical Sciences Research Center, Tehran University of Medical Sciences, Tehran, Iran

\begin{abstract}
A series of poly-functionalized tacrine-derived compounds namely 5-amino-2-phenyl- $4 \mathrm{H}$ pyrano[2,3-b]quinoline-3-carboxylates were designed and synthesized as cholinesterases inhibitors. The in vitro inhibition assay against $\mathrm{AChE}$ and $\mathrm{BuChE}$ demonstrated that most of compounds had potent AChE inhibitory with reserving potential of BuChE inhibition. Among them, compound $\mathbf{6} \mathbf{i}$ bearing a 4-(3-bromophenyl) moiety showed the most potent activity against $\mathrm{AChE} / \mathrm{BuChE}\left(\mathrm{IC}_{50} \mathrm{~S}\right.$ values of 0.069 and $1.35 \mu \mathrm{M}$, respectively). The anti-AChE activity of $6 \mathbf{i}$ was five times more than that of tacrine. The SAR study revealed that chloro/bromo substituent at ortho or meta position of the 4-phenyl ring can improve the anticholinesterase activity.
\end{abstract}

Keywords: Acetylcholinesterase; butyrylcholinesterase; Alzheimer's disease; tacrine

\footnotetext{
* Corresponding authors: E-mail address: ysarrafi@umz.ac.ir (Y. Sarrafi); E-mail address: aforoumadi@yahoo.com (A. Foroumadi), Tel.: +98 21 66954708; fax: +98 2166461178.
} 


\section{Introduction}

Alzheimer's disease (AD) is a complex neurodegenerative disorder of the brain, affecting a large number of elderly populations. AD is the most common cause of dementia and death in aging peoples [1,2]. The estimated number of patients with dementia in the world is currently expected to be 46 million rising to 131.5 million by 2050 [3].

The molecular basis of $\mathrm{AD}$ is yet to be determined but this disease is associated with a deficiency in cholinergic neurotransmission in the brain areas related to memory and learning [4]. Furthermore, $\beta$-amyloid plaques and neurofibrillary tangles are the major pathological signs in the brain of AD patients [5].

Currently, most of the drugs approved for the AD therapy are acetylcholinesterase (AChE) inhibitors including donepezil, rivastigmine, and galantamine [6-8]. These drugs are able to enhance cholinergic neurotransmission by increasing acetylcholine (ACh) level in the central nervous system [9]. Recent studies in AD patients indicated that while the AChE activity is greatly reduced in specific regions of the brain, the butyrylcholinesterase (BuChE) activity increases. Due to the involvement of BuChE in the hydrolysis of ACh, it seems the inhibition of both types of cholinesterase enzymes is essential for successful treatment of AD [10-12].

Besides normal hydrolyzing function, $\mathrm{AChE}$ also acts as a promoter of $\mathrm{A} \beta$ fibril formation $[13,14]$. It has been demonstrated AChE inhibitors that interact with the peripheral site of the enzyme could inhibit the aggregation of $\beta$-amyloid proteins [15]. However, the clinical usefulness of $\mathrm{AChE}$ inhibitors is limited, mainly due to their adverse effects. In particular, tacrine which was the first AChE inhibitor approved by FDA, has been removed from the market because of hepatotoxicity [16-18]. Accordingly, a part of attentions is focused on developing new AChE inhibitors derived from tacrine with improved activity and reduced toxicity.

Several reports have considered design of tacrine-like compounds by replacing or annulating the benzene ring in tacrine with different heterocyclic systems. Recently we introduced some pyrazolo[4',3':5,6]pyrano[2,3-b]quinolines (I) as new tacrine-based AChE inhibitors [19,20]. Maalej et al. synthesized a series of benzopyran-derived tacrines namely 7-aryl-9,10,11,12tetrahydro-7H-benzo[7,8]chromeno[2,3-b]quinolin-8-amines (II) as multi-targeted AChE inhibitors. Based on their biological results, the compounds had potent and selective inhibitory activity against AChE in the micromolar and submicromolar ranges [21,22]. 
Moreover, numerous tacrine-based compounds (III) bearing 4H-pyrano[2,3-b]quinoline-3carboxylate scaffold have been synthesized and evaluated by Marco team as acetylcholinesterase inhibitors with neuroprotective activity [23].

As seen in Fig. 1, all described compounds I-III share in the same scaffold namely tetrahydro-pyrano[2,3- $b$ ]quinoline resembling the framework of well-known drug tacrine. In continuation of our works on hetero-annulated pyrans $[24,25]$, the tetrahydro-pyrano[2,3$b$ ]quinoline structure was used as a pharmacophoric moiety in the design of new AChE inhibitors. Thus, we report here the synthesis and biological evaluation of ethyl 5-amino-2phenyl-6,7,8,9-tetrahydro-4H-pyrano[2,3-b]quinoline-3-carboxylates 6a-p. Structurally, the designed compounds 6a-p are derived from compounds III by replacing the methyl group with phenyl ring which enables the molecule to make additional $\pi-\pi$ interaction with aromatic or heteroaromatic amino acid residues of enzyme binding site. Furthermore, the attached phenyl ring in the designed compounds 6a-p is matched to benzene moiety annulated to the tetrahydro-pyrano[2,3-b]quinoline in the lead compounds II.

$<$ Fig. 1>

\section{Chemistry}

The target compounds 6a-p were synthesized in two steps as illustrated in Scheme 1. Firstly, a multi-component reaction between ethyl 3-oxo-3-phenylpropanoate (1), appropriate benzaldehyde derivative 2 and malononitrile (3) in the presence of piperdine as catalyst afforded corresponding ethyl 6-amino-5-cyano-4H-pyran-3-carboxylate derivatives 4a-p. Cyclization of compounds 4a-p with cyclohexanone by using Friedländer reaction in the presence of $\mathrm{AlCl}_{3}$ gave the final compounds 6a-p.

\section{$\langle$ Scheme 1>}

\section{Results and discussion}

The anti-cholinesterase activity of the synthesized compounds 6a-p was evaluated in vitro against $\mathrm{AChE}$ and $\mathrm{BuChE}$. The obtained results were summarized as $\mathrm{IC}_{50}$ values of tested compounds in Table 1. The well-known cholinesterase inhibitor tacrine was used as reference drug. 


\subsection{Anti-AChE activity}

A survey on the $\mathrm{IC}_{50}$ values against $\mathrm{AChE}$ revealed that all compounds had significant inhibitory activity at micro-molar and submicro-molar levels $\left(\mathrm{IC}_{50}=0.069-6.22 \mu \mathrm{M}\right)$. The 3bromo- derivative $6 \mathbf{i}$ with $\mathrm{IC}_{50}$ value of $0.069 \mu \mathrm{M}$ was found to be the most potent compound against AChE. This compound was 5-fold more potent than reference drug tacrine. It should be noted that the 2-Br, 2-Cl and 3-Cl analogs (compounds $6 \mathbf{m}, \mathbf{6 n}$ and $\mathbf{6 j}$, respectively) were also more potent than tacrine against AChE. Furthermore, the anti-AChE activity of compounds $\mathbf{6 a}, \mathbf{6 c}, \mathbf{6 f}$, and $\mathbf{6 0}$ was comparable to that of tacrine. The comparison of parasubstituted compounds 6b-g with unsubstituted congener 6a demonstrated that the introduction of substituent at the para position of 4-phenyl ring cannot improve the antiAChE activity. As observed in compound $\mathbf{6 b}$, the 4-methyl substituent significantly decreased the activity. In contrast, the insertion of bromo or chloro group at the ortho or meta position increased the inhibitory activity. Thus, compounds $\mathbf{6 i}, \mathbf{6 j}, \mathbf{6 m}$ and $\mathbf{6 n}$ showed superior activity respect to the parent compound 6a. The best result was obtained by bromo substituent at meta position. The 2,4-dichloro analog 6p was less active than 2-chloro and 4chloro derivatives (compounds $\mathbf{6 n}$ and $\mathbf{6 e}$ ). Therefore, the concurrent 2,4-substitution was not favorable.

\subsection{Anti-BuChE activity}

Based on $\mathrm{IC}_{50}$ values listed in Table $1, \mathrm{BuChE}$ enzyme was less susceptible to the target compounds compared to AChE. However, the tested compounds showed remarkable antiBuChE activity with $\mathrm{IC}_{50}$ values in the range of 1.35-74.25 $\mu \mathrm{M}$. Interestingly, the most potent compound against AChE (compound 6i) displayed the highest activity towards BuChE. Moreover, potent compounds against $\mathrm{AChE}$ (6j and $\mathbf{6 m - 0}$ ) significantly inhibited the BuChE enzyme $\left(\mathrm{IC}_{50} \mathrm{~S}=2.14-3.63 \mu \mathrm{M}\right)$. From the SAR point of view, there was similarity between BuChE and AChE. While the para-substitution on the 4-phenyl moiety diminished the activity, the presence of halogen $(\mathrm{Cl}$ or $\mathrm{Br})$ at the ortho or meta position had positive effect on BuChE inhibition. Again best result was obtained by compound 6i bearing a bromo group at the meta position.

$<$ Table 1> 


\subsection{Kinetic study of AChE inhibition}

To determine the mechanism of enzyme inhibition the kinetic study was performed using $\mathbf{6 i}$ as representative compound. The relative velocity of the enzyme was determined on three increasing concentrations of the substrate ATChI (acetylthiocholine iodide). To construct the Lineweaver-Burk plot, the enzyme velocity was measured in the presence of inhibitor (6i) at following concentrations: $0,35,70$ and $140 \mathrm{nM}$. The Lineweaver-Burke plot was then schemed using the reciprocal of velocity and substrate concentration (Fig. 2a). Based on the obtained plot, a mixed type of inhibition by compound 6i was established. Using the Lineweaver-Burk secondary plot (Fig. 2b), a $K_{\mathrm{i}}$ value equal to $48.5 \mathrm{nM}$ was obtained for compound $\mathbf{6 i}$.

$<$ Fig. 2>

\subsection{Molecular Docking}

\subsubsection{Docking study with AChE}

The ligand-protein docking was guided by AutoDock Vina (1.1.2) [26] to predict the binding poses of the ligand in the active site of AChE. The 3D coordinate of the AChE (PDB ID: 1acj) in complex with tacrine was retrieved from Protein Data Bank (PDB) at http://www.rcsb.org/pdb/home/home.do. For protein preparation, all of the non-protein atoms were removed and then minimized using OPLS3 force field (RMSD $=0.3 \AA$ ). The grid box with the size of $25 \times 25 \times 25$ was determined and the center of box was fixed on co-crystal ligand. After docking the best pose was selected for further analysis.

The molecular docking results for representative compound $\mathbf{6 i}$ showed that the ligand was well accommodated in the active site. The ligand was laid at the bottom of the active site gorge near catalytic triad. Since the chemical nature of the binding site was hydrophobic therefore the hydrophobic interactions played an important role in binding affinity. Tyr70, Asp72, Trp84, Phe290, Phe330, Tyr334, Trp432, Ile439 and Tyr442 are responsible for hydrophobic interaction with hydrophobic fragments of the ligand as depicted in Fig. 3 (All figures was prepared using Discovery Studio Visualizer 4.5 client). The central pyridine ring was stacked between two aromatic residues Trp 84 and Phe330 by making $\pi-\pi$ interaction. 
This orientation of the ligand enables it to make a hydrogen bond with His440 via amino moiety. In this position the cyclohexyl ring was well fitted in the hydrophobic pocket that located in the vicinity of catalytic triad. At the other side of the ligand that has more hydrophobic nature, the hydrophobic interactions are dominant. The 2-phenyl moiety attached to pyran ring involved in T-shaped $\pi$ - $\pi$ interaction and $\pi$-anion interaction with Tyr131 and Asp72, respectively. The pendant ethyl carboxylate was fixed by three $\pi$-alkyl interactions accompanied by hydrogen bond with Ser122. Finally the 3-bromophenyl moiety made another T-shaped $\pi-\pi$ interaction with $\operatorname{Trp} 84$.

$<$ Fig. 3>

\subsubsection{Docking study with BuChE}

Due to the significant potency of target compounds on BuChE, a ligand-protein docking was also conducted to predict the binding poses of the representative compound $\mathbf{6} \mathbf{i}$ in the active site of BuChE. The 3D coordinate of the human BuChE (PDB ID: 5LKR) in complex with $\mathrm{N}$ propargylpiperidine was retrieved from PDB. For protein preparation, all of the non-protein atoms were removed and then minimized using OPLS3 force field (RMSD=0.3 $\mathrm{A}$ ). The grid box with the size of $30 \times 30 \times 30$ was determined and the center of box was fixed on cocrystalized ligand. After docking the best pose was selected for further analysis.

As depicted in Fig. 4, compound $\mathbf{6 i}$ was well fitted in the active site cavity of BuChE. The hydrophobic and hydrogen bonds were predominant in ligand binding. The nitrogen atom of the core pyridine ring contributed in hydrogen-binding with Ser198, the key residue in the catalytic triad. The fused cyclohexane ring oriented to hydrophobic pocket composed by Val288, Leu286 and Trp231. On the other side of molecule, the $\pi-\pi$ interactions were predominant. Two phenyl rings on position 2 and 4 were involved in $\pi-\pi$ interactions with Trp82 and Tyr332 respectively.

<Fig. 4>

\subsection{Prediction of blood-brain barrier (BBB) permeability}

The BBB penetration of synthesized compounds 6a-p was predicted by using admetSAR server (http://lmmd.ecust.edu.cn:8000/predict/) [27]. The predicted data were listed in Table 
2. As seen, all compounds with the exception of nitro- derivatives $\mathbf{6 g}$ and $\mathbf{6 l}$ are BBB permeable.

$<$ Table 2>

\subsection{Neuroprotective effect against ROS-mediated cell apoptosis in differentiated PC12 cells}

The neuroprotective potential of the promising compounds $\mathbf{6 i}, \mathbf{6 j}, \mathbf{6 m}$ and $\mathbf{6 n}$ against $\mathrm{H}_{2} \mathrm{O}_{2^{-}}$ induced cell death in differentiated PC12 cells was evaluated in comparison to quercetine and tactine. The differentiated cells from PC12 neurons were pretreated with different concentrations $(1,10$ and $100 \mu \mathrm{M})$ of the compounds for $3 \mathrm{~h}$, before treatment with $\mathrm{H}_{2} \mathrm{O}_{2}(300$ $\mu \mathrm{M})$. Addition of $\mathrm{H}_{2} \mathrm{O}_{2}$ induced apoptosis in PC12 cells and then cell viability was measured after $24 \mathrm{~h}$ by using the MTT (3-(4,5-dimethylthiazol-2-yl)-2,5-diphenyltetrazolium bromide) colorimetric assay.

The obtained results for compounds $\mathbf{6 i}, \mathbf{6 j}, \mathbf{6 m}$ and $\mathbf{6 n}$ in the concentrations of 1,10 and 100 $\mu \mathrm{M}$ were compared with control group, quercetine and tacrine $(10 \mu \mathrm{M})$ and depicted in Fig. 5. As shown in Fig. 5, $\mathrm{H}_{2} \mathrm{O}_{2}$ significantly reduced the cell viability compared with negative control. The pretreatment of PC12 cells with the test compounds protected neurons against cell death. In particular, compound $\mathbf{6 n}$ could significantly protect cells at the concentration of $10 \mu \mathrm{M}(P<0.0001)$. All compounds were effective on prevention of ROS mediated cell apoptosis at concentration of $100 \mu \mathrm{M}(P<0.01)$.

\section{$<$ Fig. 5>}

\subsection{Cytotoxicity on Hepatocyts}

The cytotoxic activity of selected compounds $\mathbf{6 i}, \mathbf{6 j}, \mathbf{6}, \mathbf{6 m}$ and $\mathbf{6 n}$ was evaluated against HepG2 cell line by using MTT cell viability assay. The $\mathrm{IC}_{50}$ values of compounds were determined and compared with that of reference drug tacrine (Table 3). While compounds 6ln showed no significant cytotoxic activity against HepG2 cells $\left(\mathrm{IC}_{50} \mathrm{~S}>100 \mu \mathrm{g} / \mathrm{mL}\right)$ but compounds $\mathbf{6 i}$ and $\mathbf{6 j}$ with $\mathrm{IC}_{50}$ values of 72.8 and $50.4 \mu \mathrm{g} / \mathrm{mL}$ showed moderate cytotoxicity. However, the cytotoxicity of the promising compound $\mathbf{6 i}$ was several times less than that reference drug tacrine $\left(\mathrm{IC}_{50}=17.9 \mu \mathrm{g} / \mathrm{mL}\right)$.

$<$ Table 3> 


\section{Conclusion}

In order to find new potential entities for treatment of $\mathrm{AD}$, a series of poly-functionalized tacrine-derived compounds namely 5-amino-2-phenyl-4H-pyrano[2,3-b]quinoline-3carboxylates were designed and synthesized as cholinesterase inhibitors. The in vitro cholinesterases inhibition assay demonstrated that most of compounds had potent AChE inhibitory with reserving potential of BuChE inhibition. Among them, compound $\mathbf{6 i}$ bearing a 3-bromophenyl moiety at 4-position of the $4 H$-pyrano[2,3-b]quinoline ring showed the most potent activity against $\mathrm{AChE} / \mathrm{BuChE}\left(\mathrm{IC}_{50} \mathrm{~s}\right.$ values of 0.069 and $1.35 \mu \mathrm{M}$, respectively). The anti-AChE activity of $\mathbf{6 i}$ was five times more than that of tacrine. Furthermore, the promising compound $\mathbf{6 i}$ showed less cytotoxicity on HepG2 cells compared to tacrine. The SAR study by the modification of substituent on the 4-phenyl ring revealed that while the electron donating and electron withdrawing group are not favorable for para-position, the electron withdrawing group such as chloro and bromo at ortho or meta can improve the cholinesterase inhibition potential of compounds.

\section{Experimental}

All commercially available reagents were used without further purification. TLC was conducted on silica gel 250 micron, F254 plates. Melting points were measured on a Kofler hot stage apparatus and are uncorrected. The IR spectra were taken using Nicolet FT-IR Magna 550 spectrograph (KBr discs). All NMR spectra were recorded on Brucker $400 \mathrm{MHz}$ NMR instruments. The chemical shifts $(\delta)$ and coupling constants $(J)$ are expressed in parts per million and Hertz, respectively. The atoms numbering of the target compounds used for ${ }^{1} \mathrm{H}$ NMR data are shown in Scheme 1. Mass spectra of the products were obtained with an HP (Agilent technologies) 5937 Mass Selective Detector. Elemental analyses were carried out by a CHN-Rapid Heraeus elemental analyzer. The results of elemental analyses $(\mathrm{C}, \mathrm{H}, \mathrm{N})$ were within $\pm 0.4 \%$ of the calculated values.

5.1. General procedure for the preparation of ethyl 6-amino-5-cyano-2,4-diphenyl-4H-pyran3-carboxylate derivatives $\mathbf{4 a - p}$

A mixture of ethyl 3-oxo-3-phenylpropanoate $(\mathbf{1}, 1 \mathrm{mmol})$, aromatic aldehyde 2 (1 $\mathrm{mmol})$, malonoitrile $(3,1 \mathrm{mmol})$, piperdine (1 drop) in ethanol $(5 \mathrm{~mL})$ was refluxed for $3 \mathrm{~h}$. After 
completion of the reaction, the mixture was cooled to $0-5{ }^{\circ} \mathrm{C}$. The precipitated solid was filtered, washed with cold ethanol and dried to give compounds 4a-p.

5.2. General procedure for the preparation of ethyl 5-amino-2,4-diphenyl-6,7,8,9-tetrahydro-4Hpyrano[2,3-b]quinoline-3-carboxylate derivatives $\mathbf{6 a - p}$

Aluminum chloride $(1.5 \mathrm{mmol})$ was suspended in dry 1,2-dichloroethane $(5 \mathrm{~mL})$ at room temperature under nitrogen atmosphere. After stirring the suspension for a few minutes, the corresponding 6-amino-5-cyano-4H-pyran 4a-p (1 mmol) and cyclohexanone $(\mathbf{5}, 1.5 \mathrm{mmol})$ were added to the mixture and the reaction mixture was heated under reflux for 3-6 h. Progress of the reaction was monitored by TLC. After completion, water was added and the mixture was basified with $10 \%$ sodium hydroxide solution to $\mathrm{pH}=8-9$. The layers were separated and the aqueous layer was extracted with dichloromethane $(3 \times 15 \mathrm{~mL})$. The combined extracts were dried $\left(\mathrm{Na}_{2} \mathrm{SO}_{4}\right)$ and concentrated. Purification by column chromatography on silica gel afforded the title compounds $\mathbf{6 a - p}$.

5.2.1. Ethyl 5-amino-2,4-diphenyl-6,7,8,9-tetrahydro-4H-pyrano[2,3-b]quinoline-3carboxylate (6a)

Yield 80\%; yellow solid; mp 154-156 ${ }^{\circ} \mathrm{C} .{ }^{1} \mathrm{H}$ NMR (400 MHz, $\left.\mathrm{CDCl}_{3}\right) \delta$ : 7.49-7.47 (m, 2H, $\mathrm{H}_{\text {phenyl }}$ ), 7.44-7.39 (m, 2H, $\left.\mathrm{H}_{\text {phenyl }}\right)$, 7.38-7.35 (m, 3H, $\left.\mathrm{H}_{\text {phenyl }}\right), 7.34-7.31$ (m, 2H, $\mathrm{H}_{\text {phenyl }}$ ), 7.25-7.21 (m, 1H, $\mathrm{H}_{\text {phenyl }}$ ), 4.98 (s, $\left.1 \mathrm{H}, \mathrm{H}_{4}\right), 4.12$ (br s, 2H, $\left.\mathrm{NH}_{2}\right), 3.89-3.84\left(\mathrm{~m}, 2 \mathrm{H}, \mathrm{CH}_{2}\right)$, 2.78-2.75 (m, 2H, 2H $), 2.37-2.32\left(\mathrm{~m}, 1 \mathrm{H}, \mathrm{H}_{6}\right), 2.24-2.20\left(\mathrm{~m}, 1 \mathrm{H}, \mathrm{H}_{6}\right), 1.82-1.79(\mathrm{~m}, 4 \mathrm{H}$, $2 \mathrm{H}_{7}$ and $\left.2 \mathrm{H}_{8}\right), 0.84\left(\mathrm{t}, 3 \mathrm{H}, \mathrm{CH}_{3}, J=7.2 \mathrm{~Hz}\right) .{ }^{13} \mathrm{C} \mathrm{NMR}\left(100 \mathrm{MHz}, \mathrm{CDCl}_{3}\right) \delta: 167.14,157.87$, 154.72 , 154.16, 150.48, 143.48, 134.90, 129.35, 128.84, 128.45, 127.58, 127.29, 113.79, 107.98, 99.26, 60.29, 39.53, 32.48, 22.92, 22.58, 22.36, 13.46. Anal. Calcd for $\mathrm{C}_{27} \mathrm{H}_{26} \mathrm{~N}_{2} \mathrm{O}$ : C, 76.03; H, 6.14; N, 6.57; Found C, 76.17; H, 6.29; N, 6.71.

5.2.2. Ethyl 5-amino-2-phenyl-4-(p-tolyl)-6,7,8,9-tetrahydro-4H-pyrano[2,3-b]quinoline-3carboxylate $(\boldsymbol{6 b})$

Yield 77\%; yellow solid; mp 114-116 ${ }^{\circ} \mathrm{C}$. IR $\left(\mathrm{KBr}, \mathrm{cm}^{-1}\right) v_{\max }: 3461,3330,3211,2942,1691$, 1633, 1449, 1278, 1084. ${ }^{1} \mathrm{H}$ NMR (400 MHz, $\mathrm{CDCl}_{3}$ ) $\delta: 7.49-7.47$ (m, 2H, $\mathrm{H}_{\text {phenyl }}$ ), 7.37-7.33 (m, 3H, $\mathrm{H}_{\text {phenyl }}$ ), 7.32-7.28 (m, 2H, $\left.\mathrm{H}_{\text {phenyl }}\right), 7.14-7.10$ (m, 2H, $\left.\mathrm{H}_{\text {phenyl }}\right), 4.94$ (s, 1H, $\left.\mathrm{H}_{4}\right), 4.13$ (bs, 2H, $\mathrm{NH}_{2}$ ), 3.90-3.84 (m, 2H, $\mathrm{CH}_{2}$ ), 2.78-2.74 (m, 2H, $\left.\mathrm{H}_{9}\right), 2.36-2.32$ (m, 1H, H6), 2.31 (s, $\left.3 \mathrm{H}, \mathrm{CH}_{3}\right), 2.28-2.20\left(\mathrm{~m}, 1 \mathrm{H}, \mathrm{H}_{6}\right), 1.82-1.79\left(\mathrm{~m}, 4 \mathrm{H}, 2 \mathrm{H}_{7}\right.$ and $\left.2 \mathrm{H}_{8}\right), 0.85\left(\mathrm{t}, 3 \mathrm{H}, \mathrm{CH}_{3}, J=\right.$ $7.2 \mathrm{~Hz}) .{ }^{13} \mathrm{C}$ NMR $\left(100 \mathrm{MHz}, \mathrm{CDCl}_{3}\right) \delta: 167.19,157.75,154.69,154.01,150.48,140.50$, 
$136.94,134.95,129.56,129.32,128.85,128.30,127.58,113.76,108.11,99.43,60.27,39.08$, 32.43, 22.92, 22.59, 22.37, 21.11, 13.47. Anal. Calcd for $\mathrm{C}_{28} \mathrm{H}_{28} \mathrm{~N}_{2} \mathrm{O}: \mathrm{C}, 76.34 ; \mathrm{H}, 6.41 ; \mathrm{N}$, 6.36; Found C, 76.51; H, 6.68; N, 6.49.

5.2.3. Ethyl 5-amino-4-(4-bromophenyl)-2-phenyl-6,7,8,9-tetrahydro-4H-pyrano[2,3b]quinoline-3-carboxylate $(\boldsymbol{6} d)$

Yield 70\%; off-white solid; mp 180-182 ${ }^{\circ} \mathrm{C} .{ }^{1} \mathrm{H}$ NMR (400 MHz, $\left.\mathrm{CDCl}_{3}\right) \delta:$ 7.46-7.42 (m, $4 \mathrm{H}, \mathrm{H}_{\text {phenyl }}$ ), 7.38-7.34 (m, 3H, $\left.\mathrm{H}_{\text {phenyl }}\right), 7.30-7.28$ (m, 2H, $\mathrm{H}_{\text {phenyl }}$ ), 4.95 (s, 1H, $\mathrm{H}_{4}$ ), 4.12 (br s, $\left.2 \mathrm{H}, \mathrm{NH}_{2}\right), 3.86\left(\mathrm{q}, 2 \mathrm{H}, \mathrm{CH}_{2}, J=7.2 \mathrm{~Hz}\right), 2.71-2.78\left(\mathrm{~m}, 2 \mathrm{H}, \mathrm{H}_{9}\right), 2.37-2.33\left(\mathrm{~m}, 1 \mathrm{H}, \mathrm{H}_{6}\right)$, 2.26-2.23 (m, $\left.1 \mathrm{H}, \mathrm{H}_{6}\right), 1.83-1.78\left(\mathrm{~m}, 4 \mathrm{H}, 2 \mathrm{H}_{7}\right.$ and $\left.2 \mathrm{H}_{8}\right), 0.83\left(\mathrm{t}, 3 \mathrm{H}, \mathrm{CH}_{3}, J=7.2 \mathrm{~Hz}\right) .{ }^{13} \mathrm{C}$ NMR (100 MHz, $\left.\mathrm{CDCl}_{3}\right) \delta: 167.04,158.45,154.64,154.37,150.35,142.52,134.70,131.93$, $130.11,129.51,128.84,127.62$, 121.21, 113.90, 107.47, 98.76, 60.42, 38.84, 32.39, 22.94, 22.52, 22.31, 13.43. Anal. Calcd for $\mathrm{C}_{27} \mathrm{H}_{25} \mathrm{BrN}_{2} \mathrm{O}_{3}$ : C, 64.16; H, 4.99; N, 5.54; Found $\mathrm{C}$, $64.26 ; \mathrm{H}, 5.18 ; \mathrm{N}, 5.74$.

5.2.4. Ethyl 5-amino-4-(4-chlorophenyl)-2-phenyl-6,7,8,9-tetrahydro-4H-pyrano[2,3b]quinoline-3-carboxylate (6e)

Yield 74\%; off-white solid; mp 185-187 ${ }^{\circ} \mathrm{C} .{ }^{1} \mathrm{H}$ NMR (400 MHz, $\left.\mathrm{CDCl}_{3}\right) \delta: 7.48-7.45$ (m, 2H, $\mathrm{H}_{\text {phenyl }}$ ), 7.39-7.33 (m, 5H, $\mathrm{H}_{\text {phenyl }}$ ), 7.29-7.27 (m, 2H, $\mathrm{H}_{\text {phenyl }}$ ), 4.97 (s, 1H, $\mathrm{H}_{4}$ ), 4.13 (br s, $2 \mathrm{H}, \mathrm{NH}_{2}$ ), 3.90-3.84 (m, 2H, $\left.\mathrm{CH}_{2}\right), 2.79-2.77$ (m, 2H, H9), 2.38-2.33 (m, 1H, $\mathrm{H}_{6}$ ), 2.27$2.24\left(\mathrm{~m}, 1 \mathrm{H}, \mathrm{H}_{6}\right), 1.84-1.82\left(\mathrm{~m}, 4 \mathrm{H}, 2 \mathrm{H}_{7}\right.$ and $\left.2 \mathrm{H}_{8}\right), 0.84\left(\mathrm{t}, 3 \mathrm{H}, \mathrm{CH}_{3}, J=7.2 \mathrm{~Hz}\right) .{ }^{13} \mathrm{C} \mathrm{NMR}$ $\left(100 \mathrm{MHz}, \mathrm{CDCl}_{3}\right) \delta: 167.05,158.35,154.62,154.32,150.39,141.96,134.69,133.09$, $129.75,129.52$, 129.00, 128.84, 127.63, 113.89, 107.58, 98.83, 60.42, 38.79, 32.36, 22.93, 22.51, 22.31, 13.43. Anal. Calcd for $\mathrm{C}_{27} \mathrm{H}_{25} \mathrm{ClN}_{2} \mathrm{O}_{3}$ : C, 70.35; H, 5.47; N, 6.08; Found C, $70.57 ; \mathrm{H}, 5.54 ; \mathrm{N}, 6.27$.

5.2.5. Ethyl 5-amino-4-(4-fluorophenyl)-2-phenyl-6,7,8,9-tetrahydro-4H-pyrano[2,3b]quinoline-3-carboxylate (6f)

Yield 85\%; off-white solid; mp 178-179 ${ }^{\circ} \mathrm{C} .{ }^{1} \mathrm{H}$ NMR (400 MHz, $\left.\mathrm{CDCl}_{3}\right) \delta: 7.50-7.49$ (m, $\left.2 \mathrm{H}, \mathrm{H}_{\text {phenyl }}\right), 7.41-7.35$ ( $\left.\mathrm{m}, 5 \mathrm{H}, \mathrm{H}_{\text {phenyl }}\right), 7.00-6.95$ (m, 2H, $\left.\mathrm{H}_{\text {phenyl }}\right), 6.42$ (br s, 2H, $\mathrm{NH}_{2}$ ), 5.07 (s, 1H, $\left.\mathrm{H}_{4}\right), 3.91-3.88\left(\mathrm{~m}, 2 \mathrm{H}, \mathrm{CH}_{2}\right), 3.02-2.99$ (m, 2H, H9), 2.54-2.48 (m, 1H, H $\mathrm{H}_{6}$, 2.38$2.34\left(\mathrm{~m}, 1 \mathrm{H}, \mathrm{H}_{6}\right), 1.79-1.72\left(\mathrm{~m}, 4 \mathrm{H}, 2 \mathrm{H}_{7}\right.$ and $\left.2 \mathrm{H}_{8}\right), 0.85$ (t, $\left.3 \mathrm{H}, \mathrm{CH}_{3}, J=7.2 \mathrm{~Hz}\right) .{ }^{13} \mathrm{C} \mathrm{NMR}$ $\left(100 \mathrm{MHz}, \mathrm{CDCl}_{3}\right) \delta: 166.11,163.51,161.05,155.83,154.12,150.10,147.04,136.45$, $131.84,130.48,130.16,130.08,128.78,127.89$, 116.17, 115.95, 113.96, 110.02, 98.46, 
61.22, 37.39, 27.29, 22.66, 21.29, 20.90, 13.45. Anal. Calcd for $\mathrm{C}_{27} \mathrm{H}_{25} \mathrm{FN}_{2} \mathrm{O}_{3}$ : C, 72.96; $\mathrm{H}$, $5.67 ; \mathrm{N}, 6.30$; Found C, 72.84; H, 5.45; N, 6.54.

5.2.6. Ethyl 5-amino-4-(4-nitrophenyl)-2-phenyl-6,7,8,9-tetrahydro-4H-pyrano[2,3b]quinoline-3-carboxylate $(\mathbf{6 g})$

Yield 83\%; yellow solid; mp 184-186 ${ }^{\circ} \mathrm{C}$; IR $\left(\mathrm{KBr}, \mathrm{cm}^{-1}\right) v_{\max }: 3467,3328,3205,2933,1698$, 1643, 1451, 1298, 1238, 1090. ${ }^{1} \mathrm{H}$ NMR (400 MHz, $\left.\mathrm{CDCl}_{3}\right) \delta: 8.20-8.17$ (m, 2H, $\mathrm{H}_{\text {phenyl }}$ ), 7.63-7.59 (m, 2H, $\mathrm{H}_{\text {phenyl }}$ ), 7.48-7.45 (m, 2H, $\left.\mathrm{H}_{\text {phenyl }}\right), 7.43-7.39$ (m, 1H, $\left.\mathrm{H}_{\text {phenyl }}\right), 7.38-7.34$ (m, 2H, $\mathrm{H}_{\text {phenyl }}$ ), 5.11 (s, 1H, $\mathrm{H}_{4}$ ), 4.11 (br s, 2H, $\mathrm{NH}_{2}$ ), 3.92-3.84 (m, 2H, $\mathrm{CH}_{2}$ ), 2.79-2.78 (m, 2H, $\left.\mathrm{H}_{9}\right), 2.40-2.35\left(\mathrm{~m}, 1 \mathrm{H}, \mathrm{H}_{6}\right), 2.30-2.23\left(\mathrm{~m}, 1 \mathrm{H}, \mathrm{H}_{6}\right), 1.85-1.80\left(\mathrm{~m}, 4 \mathrm{H}, 2 \mathrm{H}_{7}\right.$ and $\left.2 \mathrm{H}_{8}\right)$, $0.83\left(\mathrm{t}, 3 \mathrm{H}, \mathrm{CH}_{3}, J=7.2 \mathrm{~Hz}\right) .{ }^{13} \mathrm{C} \mathrm{NMR}\left(100 \mathrm{MHz}, \mathrm{CDCl}_{3}\right) \delta: 166.88,159.65,154.98,154.66$, $150.84,150.10,147.04,134.47,129.76,129.27,128.87,127.68,124.09,114.10,106.63$, 98.16, 60.59, 39.21, 32.52, 22.98, 22.49, 22.27, 13.40. Anal. Calcd for $\mathrm{C}_{27} \mathrm{H}_{25} \mathrm{~N}_{3} \mathrm{O}_{5}$ : C, 68.78; H, 5.34; N, 8.91; Found C, 68.94; H, 5.54; N, 8.77.

5.2.7. Ethyl 5-amino-4-(3-methoxyphenyl)-2-phenyl-6,7,8,9-tetrahydro-4H-pyrano[2,3b]quinoline-3-carboxylate (6h)

Yield 85\%; yellow solid; mp 142-145 ${ }^{\circ} \mathrm{C}$. IR (KBr, cm $\left.{ }^{-1}\right) v_{\max }: 3461,3322,3214,2942,1690$, 1635, 1450, 1291, 1241, 1084. ${ }^{1} \mathrm{H}$ NMR (400 MHz, $\left.\mathrm{CDCl}_{3}\right) \delta: 7.49-7.47$ (m, 2H, $\mathrm{H}_{\text {phenyl }}$ ), 7.39-7.32 (m, 3H, $\left.\mathrm{H}_{\text {phenyl }}\right)$, 7.25-7.21 (m, 1H, $\left.\mathrm{H}_{\text {phenyl }}\right), 7.02-7.00\left(\mathrm{~m}, 1 \mathrm{H}, \mathrm{H}_{\text {phenyl }}\right), 6.94-6.96$ (m, 1H, $\mathrm{H}_{\text {phenyl }}$ ), 6.78-6.76 (m, 1H, $\mathrm{H}_{\text {phenyl }}$ ), 4.97 (s, 1H, $\mathrm{H}_{4}$ ), 4.16 (br s, 2H, $\mathrm{NH}_{2}$ ), 3.87 (q, $\left.2 \mathrm{H}, J=7.2 \mathrm{~Hz}, \mathrm{CH}_{2}\right), 3.76\left(\mathrm{~s}, 3 \mathrm{H}, \mathrm{OCH}_{3}\right), 2.76-2.75\left(\mathrm{~m}, 2 \mathrm{H}, \mathrm{H}_{9}\right), 2.36-2.33\left(\mathrm{~m}, 1 \mathrm{H}, \mathrm{H}_{6}\right)$, 2.25-2.21 (m, 1H, $\left.\mathrm{H}_{6}\right), 1.82-1.79\left(\mathrm{~m}, 4 \mathrm{H}, 2 \mathrm{H}_{7}\right.$ and $\left.2 \mathrm{H}_{8}\right), 0.85\left(\mathrm{t}, 3 \mathrm{H}, \mathrm{CH}_{3}, J=7.2 \mathrm{~Hz}\right) .{ }^{13} \mathrm{C}$ NMR (100 MHz, $\left.\mathrm{CDCl}_{3}\right) \delta: 167.11,160.00,157.80,154.65,154.10,150.62,145.02,134.88$, $129.78,129.36,128.82$, 127.60, 124.84, 114.48, 113.77, 112.36, 107.89, 99.12, 60.32, 55.21, 39.52, 32.42, 29.69, 22.92, 22.56, 22.36, 13.49. Anal. Calcd for $\mathrm{C}_{28} \mathrm{H}_{28} \mathrm{~N}_{2} \mathrm{O}_{4}: \mathrm{C}, 73.66 ; \mathrm{H}$, $6.18 ;$ N, 6.14; Found C, 73.84; H, 6.24; N, 6.08.

5.2.8. Ethyl 5-amino-4-(3-bromophenyl)-2-phenyl-6,7,8,9-tetrahydro-4H-pyrano[2,3b]quinoline-3-carboxylate (6i)

Yield 85\%; off-white solid; mp 218-220 ${ }^{\circ} \mathrm{C} .{ }^{1} \mathrm{H}$ NMR (400 MHz, $\left.\mathrm{CDCl}_{3}\right) \delta: 7.52-7.49$ (m, $\left.2 \mathrm{H}, \mathrm{H}_{\text {phenyl }}\right), 7.41-7.40$ (m, 1H, $\left.\mathrm{H}_{\text {phenyl }}\right), 7.40-7.37$ (m, 4H, $\left.\mathrm{H}_{\text {phenyl }}\right), 7.34-7.31$ (m, $1 \mathrm{H}$, $\left.\mathrm{H}_{\text {phenyl }}\right), 7.26-7.20$ (m, 2H, $\left.\mathrm{H}_{\text {phenyl }}\right), 4.97$ (s, $\left.1 \mathrm{H}, \mathrm{H}_{4}\right), 4.08$ (br s, 2H, $\left.\mathrm{NH}_{2}\right), 3.91-3.87$ (m, 2H, $\left.\mathrm{CH}_{2}\right), 2.82-2.79\left(\mathrm{~m}, 2 \mathrm{H}, \mathrm{H}_{9}\right), 2.40-2.33\left(\mathrm{~m}, 1 \mathrm{H}, \mathrm{H}_{6}\right), 2.32-2.48\left(\mathrm{~m}, 1 \mathrm{H}, \mathrm{H}_{6}\right), 1.87-1.84(\mathrm{~m}$, 
$4 \mathrm{H}, 2 \mathrm{H}_{7}$ and $\left.2 \mathrm{H}_{8}\right), 0.90\left(\mathrm{t}, 3 \mathrm{H}, J=7.2 \mathrm{~Hz}, \mathrm{CH}_{3}\right) \cdot{ }^{13} \mathrm{C}$ NMR $\left(100 \mathrm{MHz}, \mathrm{CDCl}_{3}\right) \delta: 166.85$, $158.68,153.46,152.34,151.42$, 147.51, 133.91, 135.17, 130.45, 129.68, 127.97, 128.41, $126.74,126.14,113.40,106.14,97.89,60.75,38.87,31.54,22.74,22.12$, 22.02, 13.74. MS, m/z (\%): $504\left[\mathrm{M}^{+}\right]$(10.7), 424 (100), 349 (85.3), 297 (76.8), 77 (26.2). Anal. Calcd for $\mathrm{C}_{27} \mathrm{H}_{25} \mathrm{BrN}_{2} \mathrm{O}_{3}$ : C, 64.16; H, 4.99; N, 5.54; Found C, 64.21; H, 5.14; N, 5.49.

5.2.9. Ethyl 5-amino-4-(3-chlorophenyl)-2-phenyl-6,7,8,9-tetrahydro-4H-pyrano[2,3b]quinoline-3-carboxylate $(\mathbf{6 j})$

Yield 78\%; yellow solid; mp 214-217 ${ }^{\circ} \mathrm{C}$; IR $\left(\mathrm{KBr}, \mathrm{cm}^{-1}\right) v_{\max }: 3416,2860,1691,1640,1451$, 1243, 1089. ${ }^{1} \mathrm{H}$ NMR (400 MHz, $\left.\mathrm{CDCl}_{3}\right) \delta: 7.50-7.47$ (m, 2H, $\left.\mathrm{H}_{\text {phenyl }}\right), 7.41-7.40$ (m, 1H, $\mathrm{H}_{\text {phenyl }}$ ), 7.39-7.36 (m, 4H, $\mathrm{H}_{\text {phenyl }}$ ), 7.33-7.30 (m, 1H, $\left.\mathrm{H}_{\text {phenyl }}\right), 7.26-7.21$ (m, 2H, $\mathrm{H}_{\text {phenyl }}$ ), 4.98 (s, 1H, $\mathrm{H}_{4}$ ), 4.10 (br s, 2H, NH 2 ), 3.93-3.87 (m, 2H, $\mathrm{CH}_{2}$ ), 2.80-2.78 (m, 2H, H9), 2.40$2.35\left(\mathrm{~m}, 1 \mathrm{H}, \mathrm{H}_{6}\right), 2.30-2.48\left(\mathrm{~m}, 1 \mathrm{H}, \mathrm{H}_{6}\right), 1.87-1.83\left(\mathrm{~m}, 4 \mathrm{H}, 2 \mathrm{H}_{7}\right.$ and $\left.2 \mathrm{H}_{8}\right), 0.87\left(\mathrm{t}, 3 \mathrm{H}, \mathrm{CH}_{3}\right.$, $J=7.2 \mathrm{~Hz}) .{ }^{13} \mathrm{C} \mathrm{NMR}\left(100 \mathrm{MHz}, \mathrm{CDCl}_{3}\right) \delta: 166.93,158.53,154.66,153.53,150.32,145.47$, 134.72 , 134.68, 130.06, 129.51, 128.57, 127.63, 127.60, 126.64, 113.91, 107.35, 98.59, $60.45,39.20,32.48,22.95,22.53,22.33,13.46 . \mathrm{MS}, \mathrm{m} / \mathrm{z}(\%): 460\left[\mathrm{M}^{+}\right]$(1.8), 349 (100), 321 (48.5), 273 (22.7), 105 (33.6), 77 (11.2). Anal. Calcd for $\mathrm{C}_{27} \mathrm{H}_{25} \mathrm{ClN}_{2} \mathrm{O}_{3}: \mathrm{C}, 70.35 ; \mathrm{H}, 5.47 ; \mathrm{N}$, 6.08; Found C, 70.52; H, 5.51; N, 6.29.

5.2.10. Ethyl 5-amino-4-(3-nitrophenyl)-2-phenyl-6,7,8,9-tetrahydro-4H-pyrano[2,3b]quinoline-3-carboxylate $(6 \mathrm{l})$

Yield 87\%; yellow solid; mp 194-196 ${ }^{\circ} \mathrm{C} .{ }^{1} \mathrm{H}$ NMR (400 MHz, $\left.\mathrm{CDCl}_{3}\right) \delta$ : 8.33-8.31 (m, 3H, $\mathrm{H}_{\text {phenyl }}$ ), 8.13-8.10 (m, 1H, $\mathrm{H}_{\text {phenyl }}$ ), 7.76-7.73 (m, 1H, $\left.\mathrm{H}_{\text {phenyl }}\right), 7.52-7.47$ (m, 3H, $\mathrm{H}_{\text {phenyl }}$ ), 7.43-7.35 (m, 3H, $\mathrm{H}_{\text {phenyl }}$ ), 5.11 (s, 1H, $\left.\mathrm{H}_{4}\right), 4.12$ (br s, 2H, NH$), 3.93-3.87$ (m, 2H, $\mathrm{CH}_{2}$ ), 2.81-2.78 (m, 2H, $\left.\mathrm{H}_{9}\right), 2.37-2.34\left(\mathrm{~m}, 1 \mathrm{H}, \mathrm{H}_{6}\right), 2.29-2.25\left(\mathrm{~m}, 1 \mathrm{H}, \mathrm{H}_{6}\right), 1.88-1.81\left(\mathrm{~m}, 4 \mathrm{H}, 2 \mathrm{H}_{7}\right.$ and $\left.2 \mathrm{H}_{8}\right), 0.85\left(\mathrm{t}, 3 \mathrm{H}, \mathrm{CH}_{3}, J=7.2 \mathrm{~Hz}\right) .{ }^{13} \mathrm{C} \mathrm{NMR}\left(100 \mathrm{MHz}, \mathrm{CDCl}_{3}\right) \delta: 166.77,159.61$, 154.99 , 154.69, 150.04, 148.38, 145.69, 134.45, 129.89, 129.73, 128.88, 127.69, 123.17, 122.44, 114.07, 106.77, 98.10, 60.62, 39.10, 32.51, 22.98, 22.48, 21.27, 13.45. Anal. Calcd for $\mathrm{C}_{27} \mathrm{H}_{25} \mathrm{~N}_{3} \mathrm{O}_{5}$ : C, 68.78; H, 5.34; N, 8.91; Found C, 68.97; H, 5.50; N, 8.71.

5.2.11. Ethyl 5-amino-4-(2-bromophenyl)-2-phenyl-6,7,8,9-tetrahydro-4H-pyrano[2,3b]quinoline-3-carboxylate (6m)

Yield 82\%; off-white solid; mp 207-208 ${ }^{\circ} \mathrm{C}$. IR $\left(\mathrm{KBr}, \mathrm{cm}^{-1}\right) v_{\max }: 3456,3320,3200,2931$, 1687, 1642, 1466, 1291, 1233, 1084. ${ }^{1} \mathrm{H}$ NMR (400 MHz, $\left.\mathrm{CDCl}_{3}\right) \delta: 7.54-7.52$ (m, 2H, 
$\left.\mathrm{H}_{\text {phenyl }}\right), 7.47-7.44$ (m, 1H, $\left.\mathrm{H}_{\text {phenyl }}\right), 7.39-7.36$ (m, 4H, $\left.\mathrm{H}_{\text {phenyl }}\right), 7.28-7.24$ (m, 1H, $\mathrm{H}_{\text {phenyl }}$ ), $7.20-7.18$ (m, 1H, $\left.\mathrm{H}_{\text {phenyl }}\right), 5.41$ (s, 1H, $\left.\mathrm{H}_{4}\right), 4.47$ (bs, 2H, $\mathrm{NH}_{2}$ ), 3.85 (q, 2H, $\mathrm{CH}_{2}, J=7.2$ $\mathrm{Hz}), 2.83-2.75\left(\mathrm{~m}, 2 \mathrm{H}, \mathrm{H}_{9}\right), 2.42-2.37\left(\mathrm{~m}, 1 \mathrm{H}, \mathrm{H}_{6}\right), 2.24-2.20\left(\mathrm{~m}, 1 \mathrm{H}, \mathrm{H}_{6}\right), 1.85-1.79(\mathrm{~m}$, $4 \mathrm{H}, 2 \mathrm{H}_{7}$ and $\left.2 \mathrm{H}_{8}\right), 0.91$ (t, $\left.3 \mathrm{H}, \mathrm{CH}_{3}, J=7.2 \mathrm{~Hz}\right) .{ }^{13} \mathrm{C} \mathrm{NMR}\left(100 \mathrm{MHz}, \mathrm{CDCl}_{3}\right) \delta: 168.54$, $160.71,155.41,153.97,151.54,142.41,134.78,132.84,131.89,130.43,129.95,129.14$, $128.74,128.95,128.47,113.85,108.14,99.14,60.54,35.68,32.51,22.98,22.64,22.52$, 13.61. MS, m/z (\%): $506\left[\mathrm{M}^{+}\right]$(2.8), 349 (100), 321 (51.9), 105 (43.6), 77 (45.5). Anal. Calcd for $\mathrm{C}_{27} \mathrm{H}_{25} \mathrm{BrN}_{2} \mathrm{O}_{3}$ : C, 64.16; H, 4.99; N, 5.54; Found C, 64.20; H, 5.14; N, 5.51.

5.2.12. Ethyl 5-amino-4-(2-chlorophenyl)-2-phenyl-6,7,8,9-tetrahydro-4H-pyrano[2,3b]quinoline-3-carboxylate (6n)

Yield 85\%; white solid; mp 118-121 ${ }^{\circ} \mathrm{C}$; IR $\left(\mathrm{KBr}, \mathrm{cm}^{-1}\right) v_{\max }: 3406,2929,1695,1637,1520$, 1346, 1244, 1086. ${ }^{1} \mathrm{H}$ NMR (400 MHz, $\left.\mathrm{CDCl}_{3}\right) \delta: 7.52-7.50\left(\mathrm{~m}, 2 \mathrm{H}, \mathrm{H}_{\text {phenyl }}\right), 7.48-7.45$ (m, $\left.1 \mathrm{H}, \mathrm{H}_{\text {phenyl }}\right), 7.40-7.33$ (m, 4H, $\left.\mathrm{H}_{\text {phenyl }}\right), 7.26-7.22$ (m, 1H, $\left.\mathrm{H}_{\text {phenyl }}\right), 7.18-7.14$ (m, 1H, $\mathrm{H}_{\text {phenyl }}$ ), 5.42 (s, $\left.1 \mathrm{H}, \mathrm{H}_{4}\right), 4.51$ (br s, $2 \mathrm{H}, \mathrm{NH}_{2}$ ), 3.91 (q, 2H, $\left.\mathrm{CH}_{2}, J=7.2 \mathrm{~Hz}\right), 2.80-2.68$ (m, $\left.2 \mathrm{H}, \mathrm{H}_{9}\right), 2.36-2.32\left(\mathrm{~m}, 1 \mathrm{H}, \mathrm{H}_{6}\right), 2.23-2.19\left(\mathrm{~m}, 1 \mathrm{H}, \mathrm{H}_{6}\right), 1.81-1.78\left(\mathrm{~m}, 4 \mathrm{H}, 2 \mathrm{H}_{7}\right.$ and $\left.2 \mathrm{H}_{8}\right)$, 0.89 (t, 3H, $\left.\mathrm{CH}_{3}, J=7.2 \mathrm{~Hz}\right) .{ }^{13} \mathrm{C} \mathrm{NMR}\left(100 \mathrm{MHz}, \mathrm{CDCl}_{3}\right) \delta: 166.72,159.13,154.58,153.91$, 150.60 , 141.51, 134.66, 131.92, 131.20, 129.56, 129.20, 128.81, 128.44, 128.12, 127.71, $113.47,107.42,98.93,60.43,35.55,32.31,22.94,22.51,22.33,13.56 . \mathrm{MS}, \mathrm{m} / \mathrm{z}(\%): 460$ $\left[\mathrm{M}^{+}\right]$(25), 349 (100), 321 (34.8), 105 (29.7), 77 (14.3). Anal. Calcd for $\mathrm{C}_{27} \mathrm{H}_{25} \mathrm{ClN}_{2} \mathrm{O}_{3}$ : C, 70.35; H, 5.47; N, 6.08; Found C, 70.53; H, 5.57; N, 6.27.

5.2.13. Ethyl 5-amino-4-(2,4-dichlorophenyl)-2-phenyl-6,7,8,9-tetrahydro-4H-pyrano[2,3b]quinoline-3-carboxylate (6p)

Yield 80\%; off-white solid; mp 134-137 ${ }^{\circ} \mathrm{C} .{ }^{1} \mathrm{H}$ NMR (400 MHz, $\left.\mathrm{CDCl}_{3}\right) \delta: 7.50-7.48(\mathrm{~m}$, $\left.2 \mathrm{H}, \mathrm{H}_{\text {phenyl }}\right), 7.38-7.32$ ( $\left.\mathrm{m}, 4 \mathrm{H}, \mathrm{H}_{\text {phenyl }}\right), 7.31-7.25$ (m, $\left.1 \mathrm{H}, \mathrm{H}_{\text {phenyl }}\right), 7.16-7.12$ (m, $1 \mathrm{H}$, $\left.\mathrm{H}_{\text {phenyl }}\right), 5.90$ (s, $\left.1 \mathrm{H}, \mathrm{H}_{4}\right), 4.39$ (br s, $\left.2 \mathrm{H}, \mathrm{NH}_{2}\right), 3.87-3.79$ (m, 2H, $\left.\mathrm{CH}_{2}\right), 2.75-2.72(\mathrm{~m}, 2 \mathrm{H}$, $\left.\mathrm{H}_{9}\right), 2.34-2.28\left(\mathrm{~m}, 1 \mathrm{H}, \mathrm{H}_{6}\right), 2.24-2.20\left(\mathrm{~m}, 1 \mathrm{H}, \mathrm{H}_{6}\right), 1.80-1.78\left(\mathrm{~m}, 4 \mathrm{H}, 2 \mathrm{H}_{7}\right.$ and $\left.2 \mathrm{H}_{8}\right), 0.79(\mathrm{t}$, $\left.3 \mathrm{H}, \mathrm{CH}_{3}, J=7.2 \mathrm{~Hz}\right) .{ }^{13} \mathrm{C} \mathrm{NMR}\left(100 \mathrm{MHz}, \mathrm{CDCl}_{3}\right) \delta: 166.81,159.94,155.28,154.07$, 150.65 , 137.10, 135.69, 135.63, 135.17, 131.34, 129.30, 128.83, 128.45, 128.15, 127.68, $113.46,103.11,95.73,60.26,36.23,32.25,22.92,22.50,22.36,13.44$. Anal. Calcd for $\mathrm{C}_{27} \mathrm{H}_{24} \mathrm{Cl}_{2} \mathrm{~N}_{2} \mathrm{O}_{3}$ : C, 65.46; H, 4.88; N, 5.65; Found C, 65.54; H, 4.68; N, 5.89. 


\subsection{Cholinesterases inhibition assay}

AChE from Electrophorus electricus (electric eel, type V-s, lyophilized powder, $\geq 1000$ units/mg protein), human $\mathrm{BuChE}$ (recombinant, expressed in goat, $\geq 500$ units/mg protein), acetylthiocholine iodide, butyrylthiocholine iodide, DTNB [5,5'-dithiobis(2-nitrobenzoic acid)], and tacrine were purchased from Sigma-Aldrich. The inhibitory activity of test compounds 6a-p against AChE/BuChE was assessed by Ellman's method [28].

Ethanol/DMSO (9:1, $1 \mathrm{~mL})$ was used as a solvent system for dissolving test compounds. The stock solution was then diluted using phosphate buffer to achieve final concentrations. A mixture of phosphate buffer $0.1 \mathrm{M}, \mathrm{pH}=8.0(2000 \mu \mathrm{L})$, DTNB $0.1 \mathrm{M}(65 \mu \mathrm{L})$, corresponding enzyme $0.2 \mathrm{U} / \mathrm{ml}(35 \mu \mathrm{L})$ and inhibitor $(50 \mu \mathrm{L})$ was incubated for $5 \mathrm{~min}$ at room temperature. Then, acetylthiocholine iodide $0.01 \mathrm{M}(10 \mu \mathrm{L})$ was added and the change of the absorbance was measured at $412 \mathrm{~nm}$ for $1 \mathrm{~min}$. The same protocol was applied for determination of BuChE activity by using butyrylthiocholine iodide as substrate. All experiments were performed on a Synergy HTX Multi-Mode Reader-BioTek.

\section{4. $\mathrm{H}_{2} \mathrm{O}_{2}$-induced cell death in PC12 cells}

All culture media and supplements were purchased from Gibco. PC12 cell line was purchased from Pasteur institute. Cells were cultivated in DMEM supplemented with $10 \%$ fetal calf serum, 5\% horse serum and antibiotics (100 units $/ \mathrm{mL}$ penicillin, $100 \mu \mathrm{g} / \mathrm{mL}$ streptomycin). To induce neuronal differentiation, PC12 cells were re-suspended using trypsin/EDTA $(0.25 \%)$ and seeded in 96 well culture plate (4000 cells/well) and cultured for 1 week in differentiation medium [DMEM $+2 \%$ horse serum+ NGF $(100 \mathrm{ng} / \mathrm{mL})+$ penicillin \& streptomycin]. To evaluate the effect of drugs on survival rate of neurons, the culture medium was changed to NGF free medium and different concentrations of test compounds $(1,10,100$ $\mu \mathrm{M})$ were applied on cells. Quercetin and tacrine $(10 \mu \mathrm{M})$ were used as positive controls. Drugs were diluted into DMEM and added to each well in the volume of $10 \mu \mathrm{L}$. Then, three hours later induction of ROS mediated apoptosis was initiated by adding the $\mathrm{H}_{2} \mathrm{O}_{2}(300 \mu \mathrm{M})$ to the medium and after $12 \mathrm{~h}$, MTT assay was performed. MTT solution $(5 \mathrm{mg} / \mathrm{mL})$ was added to each well in a volume of $10 \mu \mathrm{L}$, and $3 \mathrm{~h}$ later $100 \mu \mathrm{L}$ of the solubilization solution [10\% SDS in $0.01 \mathrm{M} \mathrm{HCl}(\mathrm{w} / \mathrm{v})]$ was added into each well. The plates were allowed to stand overnight in the incubator in a humidified atmosphere. Absorbance at $570 \mathrm{~nm}$ was 
determined for each well using an ELISA reader. Each experimental was performed in triplicates.

\subsection{Cytotoxicity assay on HepG2 cells}

The HepG2 cell line was provided from Pasture institute, Tehran, Iran. Cells were cultured at 25 mL flask in RPMI 1640 medium (Biowest, France) containing $10 \%$ Fetal Bovine Serum (Gibco, USA) and 1\% pen/strep (PAA, Austria) in saturated humidity and $5 \% \mathrm{CO}_{2}$ incubator. The cytotoxicity of compounds was studied by MTT assay. The cells were seeded in 96 well plate $\left(6 \times 10^{6}\right.$ cell per well). After $20 \mathrm{~h}$, the cells were treated with various concentrations of compounds (500, 250, 125, 60 and $30 \mu \mathrm{g}$ dissolved in DMSO). The concentration of DMSO was $2 \%$ of medium culture. After $24 \mathrm{~h}$ incubation, the RPMI 1640 medium of each well replaced with $100 \mu \mathrm{l}$ MTT solution $(0.5 \mathrm{mg} / \mathrm{mL}$ dissolved in PBS) and the plate was incubated for $4 \mathrm{~h}$ in saturated humidity and $5 \% \mathrm{CO}_{2}$ incubator. Then, $100 \mu 1$ DMSO was added to each well, gently shaken and the absorbance was read with ELISA reader at $570 \mathrm{~nm}$.

\section{Acknowledgments}

This research has been supported by grants from the Research Council of Tehran University of Medical Sciences (Grant No. 95-03-92-33171) and Iran National Science Foundation (INSF).

\section{References}

[1] A. Gella, N. Durany, Oxidative stress in Alzheimer disease. Cell Adh. Migr. 3 (2009) 8893.

[2] M.J. Prince, F. Wu, Y. Guo, L.M. Gutierrez Robledo, M. O’Donnell, R. Sullivan, et al. The burden of disease in older people and implications for health policy and practice. Lancet 385 (2015) 549-62.

[3] The Global Impact of Dementia, World Alzheimer Report 2015, Published by Alzheimer's Disease International (ADI), London. 
[4] A.V. Terry, J.J. Buccafusco, The cholinergic hypothesis of age and Alzheimer's diseaserelated cognitive deficits: recent challenges and their implications for novel drug development. J. Pharmacol. Exp. Ther. 306 (2003) 821-827.

[5] D.J. Selkoe, M.B. Podlisny, Deciphering the genetic basis of Alzheimer's disease, Annu. Rev. Genomics Hum. Genet. 3 (2002) 67-99.

[6] H.M. Bryson, P. Benfield, Donepezil. Drugs Aging 10 (1997) 234-239.

[7] L.J. Scott, K.L. Goa, Galantamine: A review of its use in Alzheimer's disease. Drugs 60 (2000) 1095-1122.

[8] R.J. Polinsky, Clinical pharmacology of rivastigmine: A new-generation acetylcholinesterase inhibitor for the treatment of Alzheimer's disease. Clin. Ther. 20 (1998) 634-647.

[9] M. Shanks, M. Kivipelto, R. Bullock, R. Lane, Cholinesterase inhibition: Is there evidence for disease-modifying effects? Curr. Med. Res. Opin. 25 (2009) 2439-2446.

[10] E. Giacobini, Cholinesterase inhibitors: new roles and therapeutic alternatives, Pharmacol. Res. 50 (2004) 433-440.

[11] R.M. Lane, S.G. Potkin, A. Enz, Targeting acetylcholinesterase and butyrylcholinesterase in dementia, Int. J. Neuropsychopharmacol. 9 (2005) 1-24.

[12] M.A. Kamal, P. Klein, W. Luo, Y. Li, H.W. Holloway, D. Tweedie, et al., Kinetics of human serum butyrylcholinesterase inhibition by a novel experimental Alzheimer therapeutic dihydrobenzodioxepine cymserine, Neurochem. Res. 33 (2008) 745-753.

[13] G.V. De Ferrari, M.A. Canales, I. Shin, L.M. Weiner, I. Silman, N.C. Inestrosa, A structural motif of acetylcholinesterase that promotes amyloid beta-peptide fibril formation. Biochemistry 40 (2001) 10447-10457.

[14] A. Alvarez, R. Alarcon, C. Opazo, E.O. Campos, F.J. Munoz, F.H. Calderon, F. Dajas, M.K. Gentry, B.P. Doctor, F.G. De Mello, N.C. Inestrosa, Stable complexes involving acetylcholinesterase and amyloid-beta peptide change the biochemical properties of the enzyme and increase the neurotoxicity of Alzheimer's fibrils, J. Neurosci. 18 (1998) $3213-3223$. 
[15] M. Bartolini, C. Bertucci, V. Cavrini, V. Andrisano beta-Amyloid aggregation induced by human acetylcholinesterase: Inhibition studies. Biochem. Pharmacol. 65 (2003) 407416.

[16] A. Nordberg, A.L. Svensson, Cholinesterase inhibitors in the treatment of Alzheimer's disease: A comparison of tolerability and pharmacology. Drug Saf. 19 (1998) 465-480.

[17] M.W. Fariss, V.R. Mumaw, L.P. Walton, Tetrahydroaminoacridine-induced apoptosis in rat hepatocytes. Toxicol. In Vitro 10 (1996) 383-393.

[18] P.B. Watkins, H.J. Zimmerman, M.J. Knapp, S.I. Gracon, K.W. Lewis, Hepatotoxic effects of tacrine administration in patients with Alzheimer's disease. JAMA 271 (1994) 992-998.

[19] M. Khoobi, F. Ghanoni, H. Nadri, A. Moradi, M. Pirali Hamedani, F. Homayouni Moghadam, S. Emami, M. Vosooghi, R. Zadmard, A. Foroumadi, A. Shafiee, New tetracyclic tacrine analogs containing pyrano[2,3-c]pyrazole: efficient synthesis, biological assessment and docking simulation study. Eur. J. Med. Chem. 89 (2015) 296303.

[20] L. Pourabdi, M. Khoobi, H. Nadri, A. Moradi, F.H. Moghadam, S. Emami, M.M. Mojtahedi, I. Haririan, H. Forootanfar, A. Ameri, A. Foroumadi, A. Shafiee, Synthesis and structure-activity relationship study of tacrine-based pyrano[2,3-c]pyrazoles targeting AChE/BuChE and 15-LOX. Eur. J. Med. Chem. 123 (2016) 298-308.

[21] E. Maalej, F. Chabchoub, M.J. Oset-Gasque, M. Esquivias-Pérez, M.P. González, L. Monjas, C. Pérez, C. de los Ríos, M.I. Rodríguez-Franco, I. Iriepa, I. Moraleda, M. Chioua, A. Romero, J. Marco-Contelles, A. Samadi, Synthesis, biological assessment, and molecular modeling of racemic 7-aryl-9,10,11,12-tetrahydro-7Hbenzo[7,8]chromeno[2,3-b]quinolin-8-amines as potential drugs for the treatment of Alzheimer's disease. Eur. J. Med. Chem. 54 (2012) 750-763.

[22] E. Maalej, F. Chabchoub, A. Samadi, C. de los Rios, A. Perona, A. Morreale, J. MarcoContelles, Synthesis, biological assessment and molecular modeling of 14-aryl10,11,12,14-tetrahydro-9H-benzo[5,6]chromeno[2,3-b]quinolin-13-amines. Bioorg. Med. Chem. Lett. 21 (2011) 2384-2388. 
[23] J.L. Marco, C. de los Ríos, M.C. Carreiras, J.E. Baños, A. Badía, N.M. Vivas, Synthesis and acetylcholinesterase/butyrylcholinesterase inhibition activity of new tacrine-like analogues. Bioorg. Med. Chem. 9 (2001) 727-732.

[24] M. Khoobi, M. Alipour, A. Sakhteman, H. Nadri, A. Moradi, M. Ghandi, S. Emami, A. Foroumadi, A. Shafiee, Design, synthesis, biological evaluation and docking study of 5oxo-4,5-dihydropyrano[3,2-c]chromene derivatives as acetylcholinesterase and butyrylcholinesterase inhibitors. J. Med. Chem. 68 (2013) 260-269.

[25] S.E. Sadat Ebrahimi, P. Ghadirian, H. Emtiazi, A. Yahya-Meymandi, M. Saeedi, M. Mahdavi, H. Nadri, A. Moradi, B. Sameem, M. Vosooghi, S. Emami, A. Foroumadi, A. Shafiee, Hetero-annulated coumarins as new AChE/BuChE inhibitors: synthesis and biological evaluation. Med. Chem. Res. In press, doi: 10.1007/s00044-016-1626-7

[26] O. Trott, A.J. Olson, AutoDockVina: improving the speed and accuracy of docking with a new scoring function, efficient optimization and multithreading. J. Comput. Chem. 31 (2010) 455-461.

[27] F. Cheng, W. Li, Y. Zhou, J. Shen, Z. Wu, G. Liu, P.W. Lee, Y. Tang, admetSAR: a comprehensive source and free tool for assessment of chemical ADMET properties. J. Chem. Inf. Model. 52 (2012) 3099-3105.

[28] G.L. Ellman, K.D. Courtney, V. Andres Jr., R.M. Feather-stone, Biochem. Pharmacol. 7 (1961) 88-95. 


\section{Captions:}

Figure 1. The structures of reported tacrine-derived AChE inhibitors I-III and newly designed compounds 6a-p

Figure 2. Kinetic of AChE inhibition by compound 6i: Left) Lineweaver-Burk plot, Right) Lineweaver-Burk secondary plot

Figure 3. (a) The best pose of the most active compound (6i) in the active site of AChE; (b) residues of the active site involved in ligand binding.

Figure 4. Illustration of compound 6i binding mode in the active site of $\mathrm{BuChE}$

Figure 5. Neuroprotective activity of selected compounds $6 \mathbf{i}, \mathbf{6 j}, \mathbf{6 m}$ and $\mathbf{6 n}$ against ROSmediated cell apoptosis in PC12 cells. The PC12 cells were treated with different concentrations (1-100 $\mu \mathrm{M})$ of the compounds for $3 \mathrm{~h}$. After $24 \mathrm{~h}$, cell viability was determined by MTT assay in the presence $\mathrm{H}_{2} \mathrm{O}_{2}$. Quercetine (Q) and tacrine (T) were used as reference drugs.

Scheme 1. Synthesis of target compounds 6a-p. Reagents and conditions: (a) piperdine, EtOH, reflux; (b) $\mathrm{AlCl}_{3}$ (1.5 equiv.), dry 1,2-dichloroethane, reflux. 
Table 1. The $\mathrm{IC}_{50}$ values of compounds 6a-p against cholinesterases in comparison with tacrine<smiles>[R]c1ccc(C2C(C(=O)OCC)=C(c3ccccc3)Oc3nc4c(c(N)c32)CCCC4)cc1</smiles>

\begin{tabular}{|c|c|c|c|}
\hline Compound & $\mathbf{R}$ & $\mathrm{IC}_{50} \mathrm{AChE}(\boldsymbol{\mu M})$ & $\mathrm{IC}_{50} \mathrm{BuChE}(\boldsymbol{\mu M})$ \\
\hline $6 \mathbf{a}$ & $\mathrm{H}$ & $0.423 \pm 0.011$ & $5.49 \pm 0.61$ \\
\hline $6 \mathbf{b}$ & 4-Me & $4.16 \pm 0.33$ & $54.1 \pm 0.67$ \\
\hline $6 c$ & 4-OMe & $0.480 \pm 0.010$ & $5.76 \pm 0.38$ \\
\hline $6 d$ & $4-\mathrm{Br}$ & $6.22 \pm 0.36$ & $74.25 \pm 0.50$ \\
\hline $6 e$ & $4-\mathrm{Cl}$ & $1.10 \pm 0.13$ & $17.6 \pm 0.35$ \\
\hline $6 f$ & $4-\mathrm{F}$ & $0.404 \pm 0.007$ & $6.1 \pm 0.51$ \\
\hline $6 \mathrm{~g}$ & $4-\mathrm{NO}_{2}$ & $0.583 \pm 0.011$ & $9.91 \pm 0.42$ \\
\hline $6 h$ & 3-OMe & $0.510 \pm 0.010$ & $7.14 \pm 0.47$ \\
\hline $6 \mathbf{i}$ & $3-\mathrm{Br}$ & $0.069 \pm 0.005$ & $1.35 \pm 0.07$ \\
\hline $\mathbf{6 j}$ & $3-\mathrm{Cl}$ & $0.228 \pm 0.006$ & $3.10 \pm 0.45$ \\
\hline $6 \mathbf{k}$ & $3-\mathrm{F}$ & $0.778 \pm 0.009$ & $11.67 \pm 0.43$ \\
\hline 61 & $3-\mathrm{NO}_{2}$ & $2.73 \pm 0.15$ & $40.95 \pm 0.64$ \\
\hline $6 \mathrm{~m}$ & $2-\mathrm{Br}$ & $0.118 \pm 0.005$ & $2.76 \pm 0.27$ \\
\hline $6 n$ & $2-\mathrm{Cl}$ & $0.153 \pm 0.006$ & $2.14 \pm 0.33$ \\
\hline 60 & $2-\mathrm{F}$ & $0.370 \pm 0.014$ & $3.63 \pm 0.39$ \\
\hline $6 p$ & $2,4-\mathrm{Cl}$ & $1.62 \pm 0.09$ & $22.68 \pm 0.56$ \\
\hline Tacrine & - & 0.356 & 0.014 \\
\hline
\end{tabular}


Table 2. The BBB permeability of compounds predicted by online admetSAR server

\begin{tabular}{lll}
\hline Compound & $\begin{array}{l}\text { Permeability } \\
\left(\mathrm{BBB}^{-+}\right)\end{array}$ & Probability \\
\hline 6a & + & 0.84 \\
$\mathbf{6 b}$ & + & 0.82 \\
$\mathbf{6 c}$ & + & 0.59 \\
$\mathbf{6 d}$ & + & 0.80 \\
$\mathbf{6 e}$ & + & 0.82 \\
$\mathbf{6 f}$ & + & 0.85 \\
$\mathbf{6 g}$ & - & 0.65 \\
$\mathbf{6 h}$ & + & 0.59 \\
$\mathbf{6 i}$ & + & 0.80 \\
$\mathbf{6 j}$ & + & 0.82 \\
$\mathbf{6 k}$ & + & 0.85 \\
$\mathbf{6 1}$ & - & 0.65 \\
$\mathbf{6 m}$ & + & 0.80 \\
$\mathbf{6 n}$ & + & 0.85 \\
$\mathbf{6 0}$ & + & 0.85 \\
$\mathbf{6 p}$ & + & 0.82 \\
Tacrine & + & 0.98 \\
\hline & &
\end{tabular}


Table 3. In vitro cytotoxicity of selected compounds on HepG2 cells in comparison to tacrine.

\begin{tabular}{ll}
\hline Compound & IC $_{\mathbf{5 0}}(\boldsymbol{\mu g} / \mathbf{m L})$ \\
\hline $\mathbf{6 i}$ & 72.8 \\
$\mathbf{6 j}$ & 50.4 \\
$\mathbf{6}$ & $>100$ \\
$\mathbf{6 m}$ & $>100$ \\
$\mathbf{6 n}$ & $>100$ \\
Tacrine & 17.9 \\
\hline
\end{tabular}




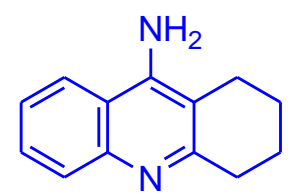

Tacrine

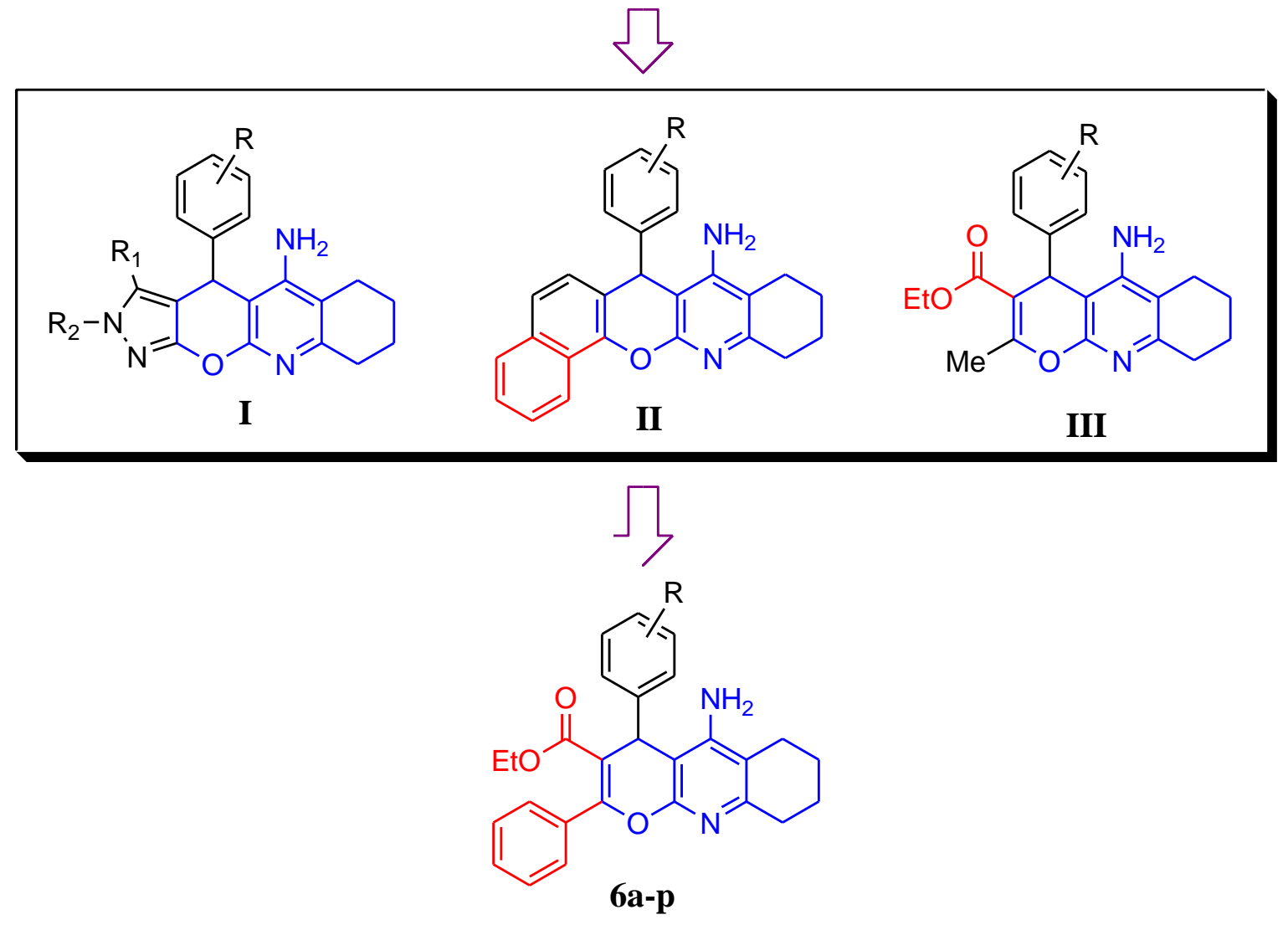

Figure 1. The structures of reported tacrine-derived AChE inhibitors I-III and newly designed compounds 6a-p 

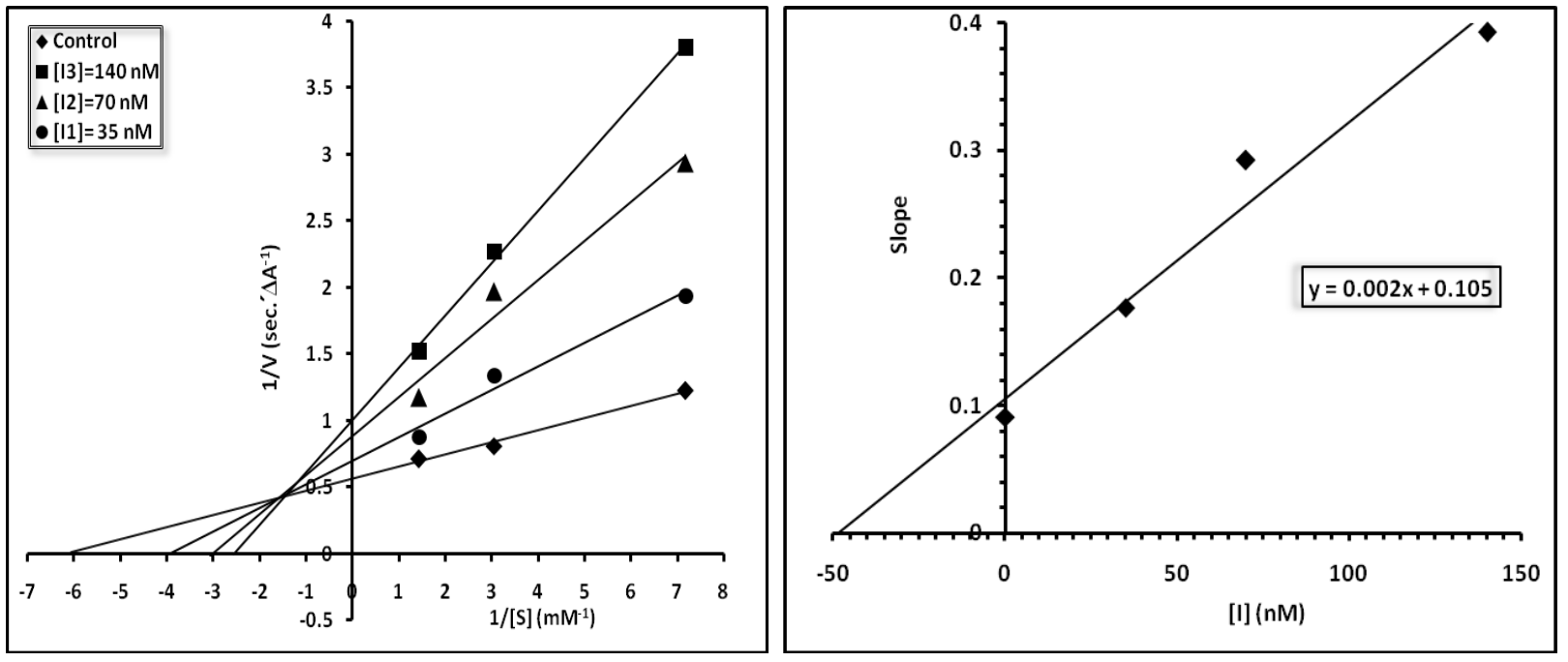

Figure 2. Kinetic of AChE inhibition by compound 6i: Left) Lineweaver-Burk plot, Right) Lineweaver-Burk secondary plot 
(a)

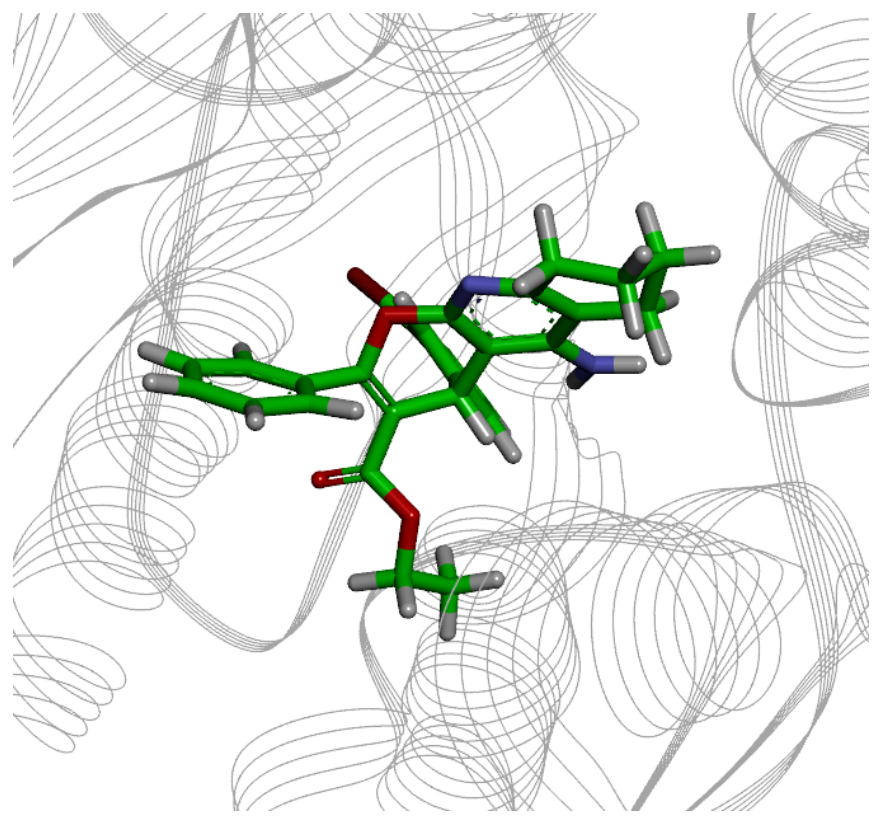

(b)

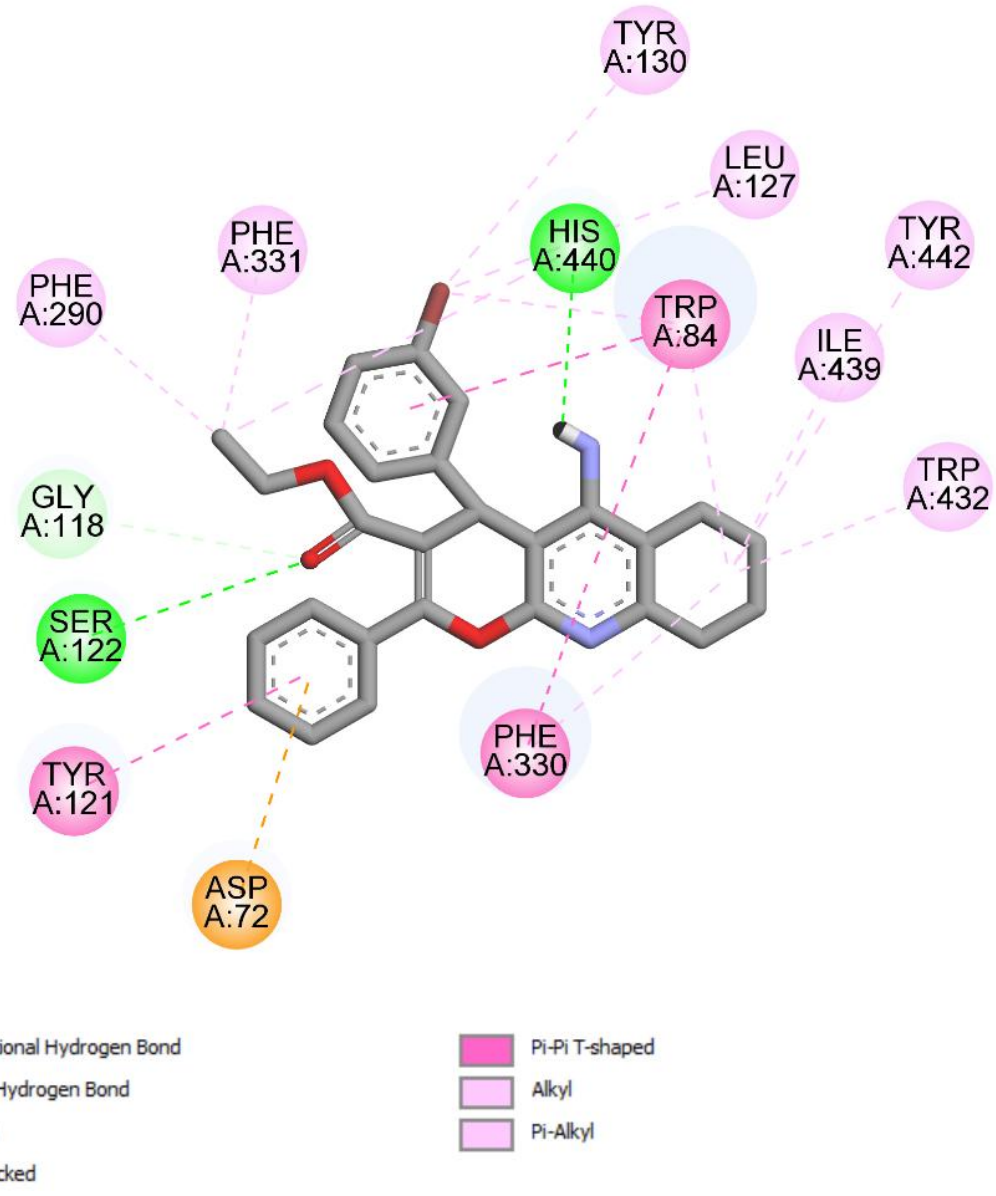

Figure 3. (a) The best pose of the most active compound (6i) in the active site of AChE; (b) residues of the active site involved in ligand binding. 


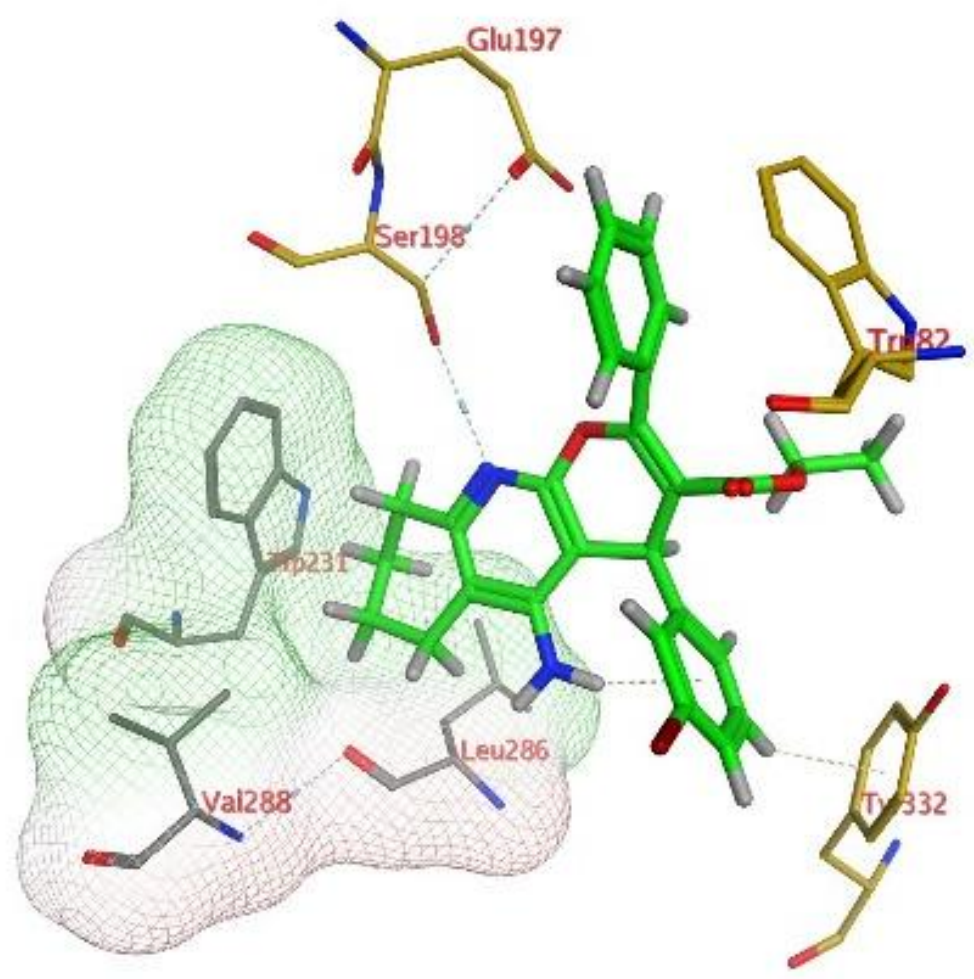

Figure 4. Illustration of compound $\mathbf{6 i}$ binding mode in the active site of $\mathrm{BuChE}$ 


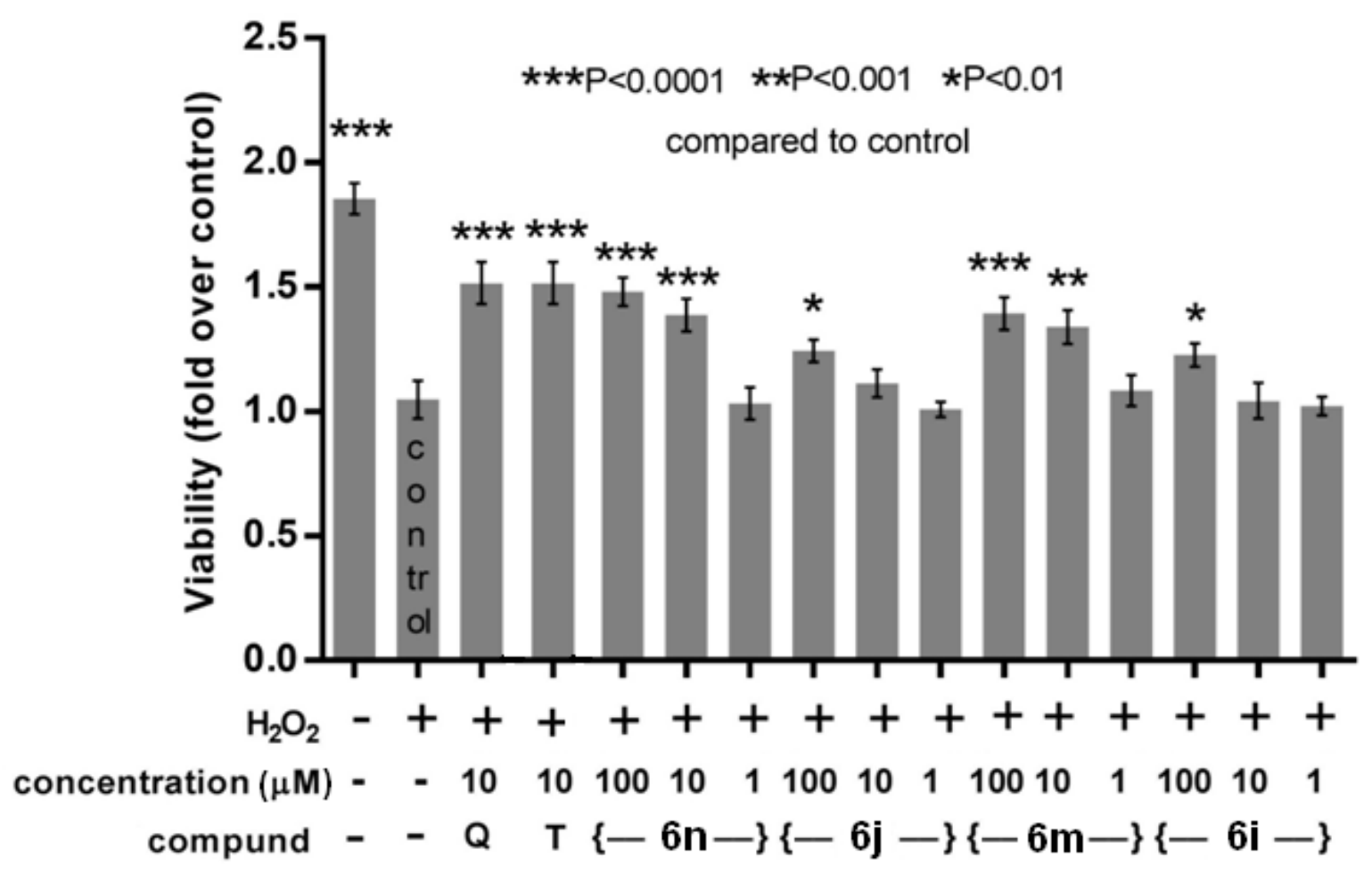

Figure 5. Neuroprotective activity of selected compounds $6 \mathbf{i}, \mathbf{6 j}, \mathbf{6 m}$ and $\mathbf{6 n}$ against ROSmediated cell apoptosis in PC12 cells. The PC12 cells were treated with different concentrations $(1-100 \mu \mathrm{M})$ of the compounds for $3 \mathrm{~h}$. After $24 \mathrm{~h}$, cell viability was determined by MTT assay in the presence $\mathrm{H}_{2} \mathrm{O}_{2}$. Quercetine (Q) and tacrine (T) were used as reference drugs. 


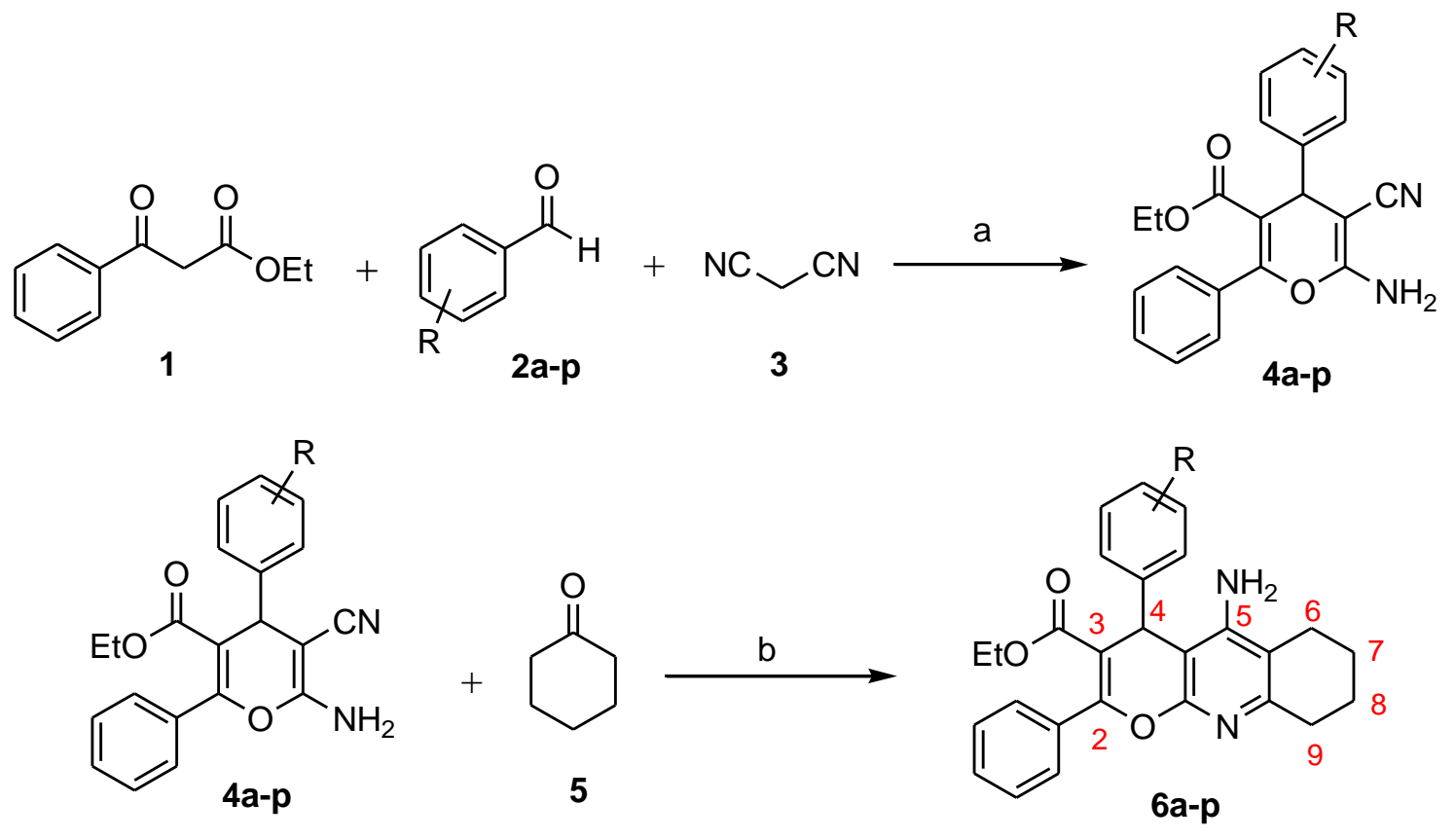

Scheme 1. Synthesis of target compounds 6a-p. Reagents and conditions: (a) piperidine, EtOH, reflux; (b) $\mathrm{AlCl}_{3}$ (1.5 equiv.), dry 1,2-dichloroethane, reflux. 


\section{Graphical Abstract}

New tacrine-derived AChE/BuChE inhibitors: synthesis and biological evaluation of 5amino-2-phenyl-4H-pyrano[2,3-b]quinoline-3-carboxylates

Mohammad Eghtedari ${ }^{\mathrm{a}}$, Yaghoub Sarrafi ${ }^{\mathrm{a}^{* *}}$, Hamid Nadri ${ }^{\mathrm{b}}$, Mohammad Mahdavi ${ }^{\mathrm{c}}$, Alireza Moradi $^{\text {b }}$, Farshad Homayouni Moghadam ${ }^{\mathrm{d}}$, Saeed Emami ${ }^{\mathrm{e}}$, Loghman Firoozpour ${ }^{\mathrm{c}}$, Ali Asadipour $^{\mathrm{f}}$, Omid Sabzevari ${ }^{\mathrm{g}}$, Alireza Foroumadi ${ }^{\mathrm{f}} \mathrm{h}_{*}$

${ }^{a}$ Faculty of Chemistry, University of Mazandaran, Babolsar, Iran

${ }^{b}$ Department of Medicinal Chemistry, Faculty of Pharmacy, Shahid Sadoughi University of Medical Sciences, Yazd, Iran

${ }^{c}$ Drug Design and Development Research Center, Tehran University of Medical Sciences,

Tehran, Iran

${ }^{d}$ Department of Cellular Biotechnology, Cell Science Research Center, Royan Institute for Biotechnology, ACECR, Isfahan, Iran.

${ }^{e}$ Department of Medicinal Chemistry and Pharmaceutical Sciences Research Center, Faculty of Pharmacy, Mazandaran University of Medical Sciences, Sari, Iran

${ }^{f}$ Department of Medicinal Chemistry, Faculty of Pharmacy and Neuroscience Research Center, Institute of Neuropharmacology, Kerman University of Medical Sciences, Kerman, Iran

${ }^{g}$ Department of Pharmacology and Toxicology, Faculty of Pharmacy, Tehran University of Medical Sciences, Tehran, Iran

${ }^{h}$ Department of Medicinal Chemistry, Faculty of Pharmacy and Pharmaceutical Sciences Research Center, Tehran University of Medical Sciences, Tehran, Iran<smiles>Nc1c2c(nc3ccccc13)CCCC2</smiles>

Tacrine

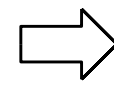<smiles>CCCCNCOCc1ccccc1</smiles>

A series of tacrine-derived compounds were synthesized as cholinesterases inhibitors. Among them, compound $\mathbf{6 i}$ with 4-(3-bromophenyl) moiety showed the most potent activity against $\mathrm{AChE} / \mathrm{BuChE}\left(\mathrm{IC}_{50} \mathrm{~S}\right.$ of 0.069 and $1.35 \mu \mathrm{M}$, respectively). 